\title{
NETWORK-BASED ASSESSMENTS OF PERCOLATION-INDUCED CURRENT DISTRIBUTIONS IN SHEARED ROD MACROMOLECULAR DISPERSIONS*
}

\author{
FENG $\mathrm{SHI}^{\dagger}$, SIMI WANG ${ }^{\dagger}$, M. GREGORY FOREST ${ }^{\dagger}, \mathrm{PETER}^{\mathrm{J}}$. MUCHA ${ }^{\dagger}$, AND \\ RUHAI ZHOU $\ddagger$
}

\begin{abstract}
Conducting high-aspect-ratio rods with 1-10 nm-scale diameters dispersed in poorly conducting matrices at extremely low, $O(1 \%)$, volume fractions induce dramatic gains in bulk conductivity at rod percolation threshold. Experimentally [Nan, Shen, and Ma, Annu. Rev. Mater. Res., 40 (2010), pp. 131-151], bulk conductivity abandons the prepercolation, linear scaling with volume fraction that follows from homogenization theory [Zheng et al., Adv. Funct. Mater., 15 (2005), pp. 627-638], and then postpercolation jumps orders of magnitude to approach that of the pure rod macromolecular phase as predicted by classical percolation theory [Stauffer and Aharony, Introduction to Percolation Theory, CRC Press, Boca Raton, FL, 1994]. Our aim here is to use the orientational probability distribution functions from kinetic Brownian rod dispersion flow codes [Forest, Wang, and Zhou, Rheol. Acta, 44 (2004), pp. 80-93] to generate physical three-dimensional (3D) nanorod dispersions, followed by graph-theoretic algorithms applied to each realization to address two practical materials science questions that lie beyond the above theoretical results. How does bulk conductivity scale in the presence of anisotropy induced by shear film flow at and above rod percolation threshold? What are the statistical distributions of current within the rod phase? Our techniques reveal a robust exponential, large current tail of the current distribution above percolation threshold that persists over a wide range of shear rates and volume fractions; the exponential rates are spatially anisotropic, with different scaling in the flow, flow gradient, and vorticity axes of the film. The second moment of the computed current distributions furthermore captures and reproduces the bulk conductivity scaling seen experimentally. These results extend the scaling behavior for the classical setting of 3D lattice bond percolation [Shi et al., Multiscale Model. Simul., 11 (2013), pp. 1298-1310] to physical 3D nanorod dispersions with random centers of mass and shear-induced anisotropy in the rod orientational distribution.
\end{abstract}

Key words. nanorod dispersions, percolation, anisotropy, scaling laws, structure-property relationships, current distribution

AMS subject classifications. 82D $30,82 \mathrm{~B} 43,82 \mathrm{~B} 80,76 \mathrm{~A} 05$

DOI. $10.1137 / 130926390$

1. Introduction. Nanorod (nm-scale diameter and large aspect ratio) dispersions in poorly conducting matrices possess remarkable macroscopic property enhancements when there is percolation in the rod phase. Analogous mechanical property enhancements are observed in nanorod-reinforced materials [37] and furthermore in diverse biological materials, including spider silk and many instances of fibrillar protein assemblies (see Veerman et al. [49] and references therein). While transport properties at percolation threshold are well understood [21, 46], we are interested in sheared thin films of nanorod dispersions with two additional features of materials engineering relevance: volume fractions above percolation threshold for property stability, and anisotropy in the particle phase due to processing history $[28,38,52]$ that is inherited by all properties concentrated on the particle phase. Each of these features

*Received by the editors June 26, 2013; accepted for publication (in revised form) December 23, 2013; published electronically March 4, 2014. This work was partially supported by ARO 12-60317MS, AFOSR FA9550-12-1-0178, NSF DMR-1122483, DMS-1100281, and DMS-0645369.

http://www.siam.org/journals/mms/12-1/92639.html

${ }^{\dagger}$ Department of Mathematics, University of North Carolina at Chapel Hill, Chapel Hill, NC 27599-3250 (bill10@uchicago.edu, wangsimi@live.unc.edu, forest@unc.edu, mucha@unc.edu).

${ }^{\ddagger}$ Department of Mathematics, Old Dominion University, Norfolk, VA 23529 (rzhou@odu.edu). 
moves anisotropic nanorod composite films out of the universality class of equilibrium percolation theory. Given the extensive literature on rod ensembles with isotropic or posited anisotropic equilibrium orientational distributions $[4,2,3,5,8,7,14,23,36]$, we are interested in how the threshold scaling behavior, widely used for guidance in materials engineering, is either perturbed or potentially strongly modified for sheared nanorod composites above but still near percolation threshold.

Unlike sheared carbon nanotube dispersions [6, 28, 38], nanorod orientational distributions are governed by rod rotational Brownian motion and nonlocal excluded volume interactions coupled to shear flow. Thus one should evaluate properties on the basis of statistical distribution functions of the rod ensemble, which the authors $[17,18]$ have previously studied in detail from the kinetic theory of Doi [13] and Hess [26]. Homogenization theory $[34,53,19]$ (based on volume and ensemble averaging of kinetic distribution functions) yields accurate, anisotropic bulk conductivity tensors in sheared nanorod dispersions below percolation thresholds, where the scaling is linear in rod volume fraction. These results fail to account for percolation in the rod phase and thereby miss the dramatic power-law scaling in conductivity enhancements at and above percolation threshold. Our goal here is to extend the homogenization results $[34,53,19]$ below threshold to explore the scaling behavior above threshold, relying on precomputed kinetic orientational distributions of sheared nanorod dispersions. To do so, we introduce graph-theoretical methods that detect and determine consequences of percolation in the rod ensemble. We also note that boundary confinement in thin films can generate significant spatial gradients in the nanorod orientational distribution $[40,44,29]$, yet in this work we focus first at the cubic micron scale where spatial homogeneity can be safely assumed (the so-called monodomain scale).

For nanometer-scale particle composites, relatively little is known about performance properties within the particle phase due to a lack of experimental resolution at scales bridging single nanorods to the bulk. We focus on current distributions within the particle phase in this paper, highlighting multiresolution, statistical tools that can fill the void in measurements. Previous predictions of current distributions, and in particular the small current tails and the scaling of the largest current in the network, have been limited to lattice resistor networks $[12,47,11,15,16,41]$. The lack of progress on multiscale transport properties in physical nanorod dispersions may be attributed both to the lack of sufficient experimental results on local properties and to the notion that lattice percolation and continuum percolation belong to the same universality class. In any case, the nonequilibrium and highly anisotropic features of sheared nanorod dispersions violate assumptions of classical percolation theory at criticality. Classical percolation theory explains the critical bulk threshold behavior in model systems, but very little is known about the property implications of several features studied herein: the scaling behavior above threshold (does it return to linear scaling with volume fraction?); the influence of shear processing (anisotropy of the particle phase); multiscale features of the current carrying rods (e.g., the fraction of rods with nonzero current, the spatial morphology of the current carrying rods); and the distribution of currents (especially the large current tail) supported by the nanorod ensemble.

So motivated, we develop multiscale electrical property metrics based on percolation in the particle phase that are reflective of the statistical properties and anisotropy of sheared Brownian nanorod dispersions. We focus on material systems with three distinctive properties: the nanorods are highly conducting relative to the matrix (cf. $[37,39,51]$ ); the rod orientational distribution may be weakly or strongly anisotropic, induced by shear flow [25, 24]; and the rod phase has macroscopic per- 
colating clusters [54]. The first property can be relaxed with a significant increase in computational complexity of the linear electrical property transport solve. Here we treat the matrix as a perfect insulator and contacts between rods as perfectly conductive. Contact resistance is important in electrical conductivity; while various models have been studied, a clear picture of the effect of contact resistance has yet to emerge $[9,30,1]$. Our model can be easily extended to incorporate contact resistance, albeit with a significant increase in the computational cost. The effect of contact resistance on the conductivity has been studied systematically in [31], and they found that the effective conductivity exponent varies in a narrow band (less than 10\%) within the critical exponent in percolation theory for a wide range of contact resistance. We note, however, that while contact resistance influences the conductivity of individual samples, the scaling behavior of conductivity and the exponents are less sensitive to contact resistance. A future study of contact resistance in DC and AC electrical conductivity of nanorod composites is worthwhile. The latter two properties, however, distinguish nanoscale rod composites for which existing property assessments are inadequate for realistic particle number densities and the number of realizations required for reasonable statistics, even at fixed rod volume fraction and Peclet number ( $P e$, shear rate normalized by rotational relaxation rate of the Brownian rods). Buxton and Balazs [10] are quite close in spirit to our work, whereas their model focuses on polymer-nanorod surface chemical interactions and our approach is amenable to hydrodynamics of nanorod dispersions. We utilize a network representation and graph algorithms to significantly reduce the transport property solve, and indeed to remove numerical error in the small current tail of the current distribution across the network. Similar network-based methods with fewer rod particles are used in the study of dielectric properties [43] and topology of the composite [42] by Simoes, Vaia, and collaborators, which motivated our approach.

We outline our method as a series of steps in section 2, referring to original publications for details of our previous results that are employed here. Then we go into detail in section 3 about the new steps involving property metrics based on the network representation of percolating components. The details for numerical simulations including the values of parameters are summarized in section 4 , followed by results on the bulk conductivities and current distributions across the percolation phase diagram.

2. Methods. Here we study Brownian nanorod dispersions where contact percolation occurs well below the nematic transition (see [54], for example). Externally imposed shear induces anisotropic rod orientations which are reflected in the local and bulk properties carried by anisotropic percolating paths. Modeling single-particle electrical response by effective resistance proportional to path length, we statistically assess multiresolution (local and bulk) electrical properties of a highly conducting rod particle phase dispersed in a relatively very poorly conducting matrix phase.

Our first step is to calculate the rod orientational probability distribution function (PDF) of a sheared nanorod dispersion by numerically solving the Doi-HessSmoluchowski equation which takes into account the effects of Brownian motion and nonlocal excluded volume interactions, as well as imposed shear. We refer the reader to Forest, Wang, and Zhou [18] for the kinetic theory and attractor phase diagrams of the nanorod orientational distributions versus rod volume fraction $\theta$ and normalized shear rate or Peclet number $P e$. These orientational distributions arise from imposed simple shear with a presumed rapid quench of rod microstructure. For each $\theta$ and $P e$ we compute the kinetic distribution function, thereby creating a database of distribu- 
tions across the $(P e, \theta)$ parameter space. (Since it turns out that percolation in the rod phase occurs at volume fractions well below the nematic transition, we focus this study at volume fractions where the unsheared stable equilibrium is isotropic [54].)

The second step populates Monte Carlo (MC) samples of three-dimensional (3D) sheared nanorod dispersions in a cubic box of length $L$, self-consistent with the orientational PDF at each fixed $(P e, \theta)$, as in Zheng et al. [54]. The nanorod centers of mass are randomly distributed in space (consistent with a fluid dispersion), with the orientation of each rod independently drawn from the precomputed PDF. This protocol yields a statistically uniform concentration of nanorods with an ensemble orientational distribution consistent with the Doi-Hess kinetic theory. Previous studies on "stick systems" with posited anisotropic orientational distributions have explored how anisotropy in the rod orientational distribution induces property anisotropy $[4,7,14]$. The property anisotropy in the present study, as in our earlier studies of homogenized effective property tensors, follows from self-consistency of the nanorod orientational PDF with a flow field (shear $[19,53,54]$ or extensional [55]), rod aspect ratio, and volume fraction. The present study, however, extends these previous results by utilizing the precomputed full orientational PDF to populate dispersions and deduce multiscale properties, whereas our earlier work utilized only second and fourth moments of the nanorod PDF together with homogenization formulas to deduce bulk, effective property tensors. The present study furthermore makes direct contact with our earlier work by taking low moments of the multiscale electrical property distribution functions to reproduce bulk, effective property tensors. On bulk scales much greater than the micron scale of our computations, spatial gradients in the PDF become important, which we do not address in this paper. Furthermore, particle-particle intersections are allowed in our soft core protocol, but this overlap volume is extremely small at the volume fractions considered here. Thus we do not introduce particle-particle collisions in either the flow code or the algorithm to populate 3D dispersions. Contrary to the early stages of nanorod composite engineering, today there are many techniques for dispersing nanorods statistically uniformly in space $[35,22,20]$. The rods are modeled as cylinders of length $l$ and diameter $d$ with two spherical caps, representing monodisperse soft-core spheroids. Since we do not check for overlap between particles in this step, the Balberg formula [2] is adopted to determine the number $N$ of rods for a given rod volume fraction $\theta$ :

$$
N=\frac{\ln (1-\theta)^{-1} L^{3}}{V}
$$

where $V$ is the volume of a single rod. For rods that are partially out of the box, periodic boundary conditions are applied so that the correct rod volume fraction is achieved.

In the third step, we extend the algorithm in Zheng et al. [54] to identify the percolating network across a specified direction (first the flow and then the flow gradient and vorticity directions) of the simulated sample. We do so by introducing a network representation of the rod ensemble and utilizing network algorithms to identify and restrict to the percolating paths (as described below). One can solve the whole resistor network problem by any standard solver, such as SPICE [23]. However, our a priori elimination filters many zero-current rods; there may be "dangling" clusters connected to the percolating paths that are not filtered here as we pragmatically balance computational impacts, as further demonstrated below. We then solve the corresponding resistor network problem, restricted to the percolating components, which significantly reduces the size of the system and improves numerical 

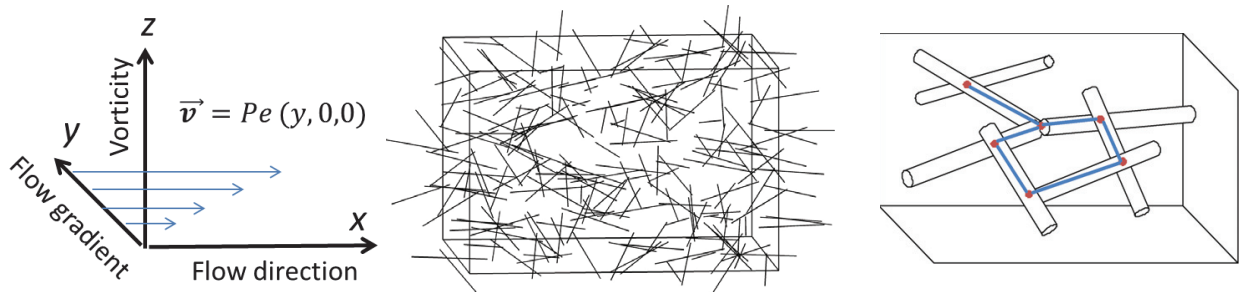

FIG. 1. Left: An illustration of the imposed shear flow with velocity field: $\vec{v}=\operatorname{Pe}(y, 0,0)$; i.e., the direction of flow is along the $x$ axis, with the $y$ axis the flow gradient direction and $z$ the vorticity direction. Center: A randomly oriented $(P e=0)$ dispersion of aspect ratio 50 rods at volume fraction .0008. The box is four times the rod length. Right: An illustration of the network representation of a few rods. Nodes are contact points of rods (the solid red dots), and edges are the effective conductances between contact points (the solid blue lines).

precision especially in the highly sensitive small current tail of the current distribution. These steps are carried out for many MC realizations at each point across the $(P e, \theta)$ phase diagram, generating a database of electrical properties superimposed onto the dimensional percolation phase diagram (as we will see in the results below in Figure 2 (right)). We then perform statistical analysis of this database that describes electrical properties in several ways, including visual depictions.

3. Network model. Every MC realization of a 3D nanorod dispersion is mapped to an undirected weighted network to study its linear DC electrical response. Recall that each MC realization distributes rods uniformly in space with orientations drawn from the specified single-particle orientational distribution. Electrically conducting contact between rods is assumed wherever rods "effectively" are in contact, that is, the minimum distance between any two rod axes is less than or equal to one rod diameter. This distance threshold of one rod diameter is a tunable parameter in our method, strongly related to electrical conductivity and resistivity properties of the rods. We have explored other distance thresholds, which has a quantitative but not qualitative influence on the results. Studies of resistive switching in nanorod composites are strongly related [50].

To study percolation and conductance along each of the three physical dimensions, (perfectly) conducting plates are assumed at the two opposite faces of the box orthogonal to the specified dimension, corresponding to imposing a voltage drop across that dimension, with all intersections between rods and the selected boundary taken to be conducting. Working nondimensionally, we treat each rod to be a conductor with unit conductivity. Then the conductance of a full rod is equal to its cross-sectional area divided by its length. For the purposes of the present model, we treat the matrix/solvent as a perfect insulator, noting that the typical ratio of conductivities is many orders of magnitude $[37,39,51]$. Every node in the corresponding electrical network represents one of the points of electrical contact between two rods or with a conducting plate, with weighted edges specified by the effective conductance between two contacts, inversely proportional to the corresponding distance along the rod connecting the two contact points (Figure 1 (right)), as represented by the (symmetric) adjacency matrix $\boldsymbol{A}$ :

$$
A_{i j}=\left\{\begin{array}{cl}
w_{i j} & \text { if node } i \text { and node } j \text { are connected } \\
0 & \text { otherwise }
\end{array}\right.
$$

with conductances $w_{i j}=s / d_{i j}$ given by the rod cross-sectional area, $s$, and the 

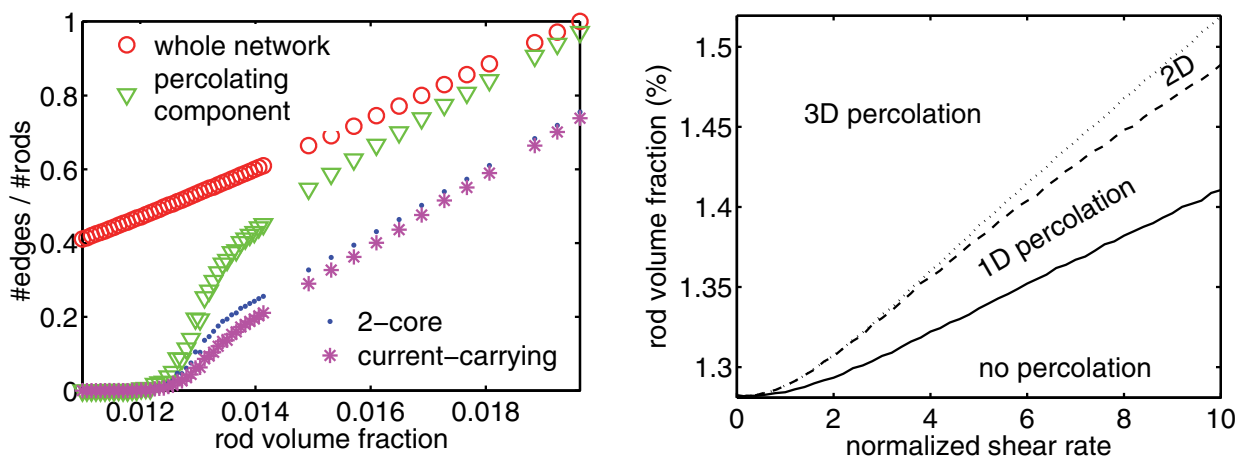

FIG. 2. Left: The average number of edges in the rod contact network (circle), in the percolating components (triangle), in the plate-constrained 2-core (dot), and in the current-carrying paths (star), normalized by the total number of rods at each rod volume fraction. Rods are isotropically oriented $(P e=0)$. Thus restriction to the plate-constrained 2-core filters approximately $90 \%$ of the rods near percolation threshold $\theta_{c} \doteq 1.3 \%$, therefore reducing the problem to $10 \%$ of its original size. Right: Percolation phase diagram with anisotropic percolation thresholds in the 2-parameter space of $(P e, \theta)$ (cf. Figure 4 in [54]). The solid, dashed, and dotted curves correspond to the percolation thresholds in the $x, y$, and $z$ directions, respectively.

distance between node $i$ and node $j, d_{i j}$. To investigate electrical conductivity in a specified direction, the conducting end plates placed on the corresponding opposite faces are each represented by a node, connected to one another through an external source.

Assuming the applied voltage is less than the dielectric breakdown strength and ignoring all inductance and electron tunneling effects, those clusters isolated from the external source will not be charged, and hence only clusters containing the two plate nodes (i.e., a connected component containing all percolating paths) need to be considered. For each realized network obtained above, we use a Dulmage-Mendelsohn decomposition of the adjacency matrix to efficiently identify the connected components that contain the two plates. The next key ingredient in our calculation procedure is the plate-constrained 2-core- defined here as the connected subgraph containing both boundary plates with degree at least two in the subgraph. This plate-constrained 2-core captures all edges that potentially carry nonzero current in the posed problem. This preprocessing step provides two significant advantages: 1. Restriction to the plate-constrained 2-core filters approximately $90 \%$ of the edges near percolation threshold, therefore reducing the linear system to $10 \%$ of its original size (e.g., see the reduction at volume fraction 1.3\% in the left panel of Figure 2). 2. By a priori elimination of all edges not in the plate-constrained 2-core, we remove a vast fraction of exactly zero-current edges from the numerical simulation of the linear system, thereby improving numerical precision overall, and in particular improved resolution of the small current tail. Therefore, we pass from the whole network to this plate-constrained 2-core and, by slight abuse of terminology, we continue to represent its adjacency matrix by $\boldsymbol{A}$. More stringent reduction within single percolating paths could be achieved by application of further graph-theoretic calculations, but such further reductions are deferred for the present results. We note that this result already informs that while a given volume fraction is necessary to statistically guarantee percolation, in fact on the order of $10 \%$ of the nanorods participate in the property gains.

The linear electrical response of this reduced network is given by Kirchoff's law 
(see, e.g., Strang [48]) $\boldsymbol{L} \boldsymbol{v}=\boldsymbol{f}$, where $\boldsymbol{v}$ is a vector indicating the voltage at each node, $\boldsymbol{f}$ is a vector consisting of the net current going out of each node, and $\boldsymbol{L}$ is the graph Laplacian matrix of the network. $\boldsymbol{L}$ is related to the adjacency matrix $\boldsymbol{A}$ by $\boldsymbol{L}=\boldsymbol{D}-\boldsymbol{A}$, with $\boldsymbol{D}$ a diagonal matrix containing the strength of each node $\left(D_{i i}=\sum_{j} A_{i j}\right)$. For all internal nodes $i, f_{i}=0$, while $f_{\text {sink }}=-f_{\text {source }}=I$ at the two nodes representing the source and sink at oppositely facing end plates.

The bulk conductance is the ratio of the external current to the obtained voltage drop across the two plate nodes, with bulk conductivity $\sigma$ following by multiplying by the box's length $L$ and dividing by its cross-sectional area $L^{2}$. That is, the bulk conductivity characterizes the macroscopic relationship between the total current passing between the two plates and the associated voltage drop. Naturally, if the two virtual nodes are not connected by a percolating path, the bulk conductivity in this model is zero. The current distribution inside the network also follows as part of this calculation. By Ohm's law, the current on an edge is equal to the voltage drop between its two endpoints multiplied by its effective conductance.

\section{Results and discussions.}

4.1. MC generation of physical 3D sheared nanorod dispersions. For each fixed $(P e, \theta)$ we create a physical 3D realization of the nanorod dispersion by drawing from the kinetic orientational distribution. Next we assess the corresponding electrical transport in each of the three spatial dimensions for each realization, obtaining the bulk conductivity for each realization and the underlying distribution of edge (rod) currents per realization. At the same $(P e, \theta)$, we generate $1000 \mathrm{MC}$ realizations and then average over all these 1000 realizations to obtain a statistical description of the electrical properties of a material volume of linear dimension 0.5 microns at each point $(P e, \theta)$ of the phase diagram of Figure 2 (right). Below we will make contact with the literature on bulk or mean properties first, then exploit our methods to drill into the multiscale properties in the rod phase, and finally show that the key bulk properties that are experimentally observed are consequences of (that is, they are predicted by) our more detailed multiscale metrics.

The imposed flow is pure shear along the $x$ axis, with the $y$ axis the flow gradient direction and $z$ the vorticity direction (see the left panel in Figure 1). For the present results, we consider a model system of typical nanorods which are $1 \mathrm{~nm} \times 50 \mathrm{~nm}$, dispersed in an $L \times L \times L$ cubic domain. For consistently comparing results at different parameter values below, we choose the box length $L$ to be 10 times the rod length (containing approximately 40,000-70,000 rods for the range of the volume fractions studied in this paper), except for the finite size scaling analysis where we consider the effect of different box lengths (section 4.3.2).

\subsection{Bulk conductivity.}

4.2.1. Dimensional percolation. At each shear rate $P e$, we calculate the critical rod volume fraction for percolation $\theta_{c}(L)=\int \theta d R_{L}$ (i.e., the effective percolation threshold as defined in [45] for a finite system with linear size $L$ ), where the percolating probability, $R_{L}(\theta)$, is approximated by the fraction of percolated samples out of 1000 realizations. We then plot the effective percolation thresholds for each of the three physical dimensions against shear rate $P e$ in the right panel of Figure 2 and reproduce the percolation phase diagram in Figure 4 of Zheng et al. [54] over a smaller domain but with higher resolution. As previously described, alignment of nanorods decreases the probability of contact, thus increasing the percolation thresholds, and the influence of shear on percolation threshold depends on the direction being consid-

Copyright (c) by SIAM. Unauthorized reproduction of this article is prohibited. 

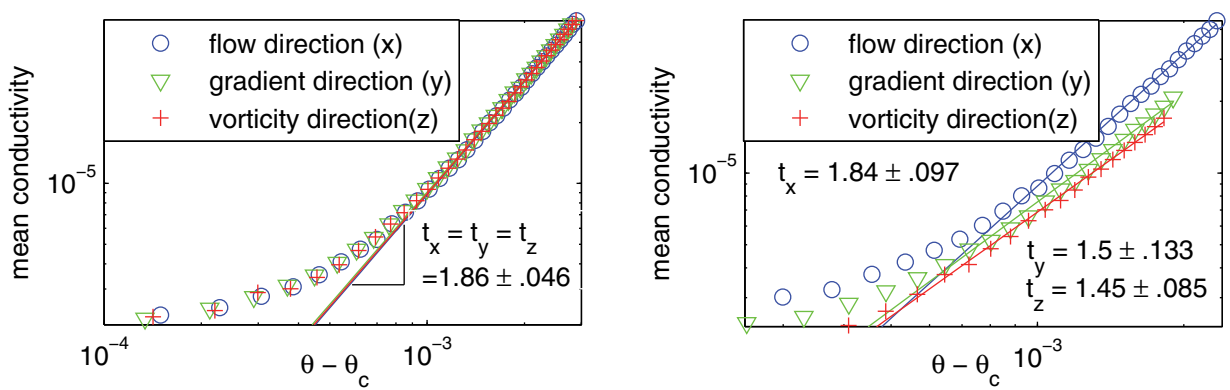

FIG. 3. Scaling of the mean conductivity $\sigma$ in each of the three directions against rod volume fraction $\theta-\theta_{c}$ for isotropic $(P e=0)$ dispersions (left) and sheared $(P e=5)$ dispersions (right). The power-law scaling of conductivity persists under shear, while the effective scaling exponent is anisotropic for Pe $>0$. Each data point is the average of 1000 realizations in a cubic box of linear dimension 0.5 microns (10 times the nanorod length).

ered, resulting in the observed dimensional percolation contrasts. In the right panel Figure 2 there is no percolation (statistically) in the region below the solid curve; percolation appears in the flow $(x)$ direction in the region between the solid curve and the dashed curve; in the region between the dashed curve and the dotted curve, percolation emerges in the flow gradient $(y)$ direction in addition to the flow $(x)$ direction; above the dotted curve, percolation spans all three dimensions. We note that the percolation thresholds appear to be linearly dependent on the normalized shear rate $P e$ over most of the range of Figure 2 (right).

4.2.2. Anisotropic conductivity. To elucidate the effect of shear on the scaling of conductivities, we first take two slices in the right panel of Figure 2 at $P e=0$ and $P e=5$. Figure 3 plots the mean conductivity $\sigma$ in each of the three directions against rod volume fraction $\theta-\theta_{c}$ for isotropic $(P e=0)$ dispersions (left) and sheared $(P e=5)$ dispersions (right). Power laws are fitted to the data, and the effective conductivity exponents are shown. Figure 3 demonstrates that the power-law scaling of conductivity $\sigma$ persists under shear with effective scaling exponents along each physical axis tuned by the shear rate Pe. Therefore we propose a general scaling form with shear:

$$
\sigma \sim\left(\theta-\theta_{c}(P e)\right)^{t(P e)}
$$

where the effective scaling exponent $t(P e)$ varies with the direction in physical space; i.e., $t_{x}(\mathrm{Pe}), t_{y}(\mathrm{Pe}), t_{z}(\mathrm{Pe})$ are distinct. Here we focus on the three physical directions: flow direction $(x)$, flow gradient direction $(y)$, and vorticity direction $(z)$. At each normalized shear rate $P e$, we fit (4.1) to the mean conductivity in each direction as in Figure 3 and plot the effective scaling exponents in Figure 4 against $P e$. From numerical fits of a quadratic function to the data in Figure 4, to the leading orders, the effective conductivity exponents $t(P e)$ scale with $P e$ approximately as

$$
\begin{aligned}
t_{x} & \approx 1.83+0.053 P e-0.010 P e^{2}, \\
t_{y} & \approx 1.83+0.018 P e-0.017 P e^{2}, \\
t_{z} & \approx 1.83+0.020 P e-0.018 P e^{2} .
\end{aligned}
$$

The quality of this fit to the numerically simulated data clearly indicates that the effective scaling exponents vary continuously with $\mathrm{Pe}$ and become anisotropic at first 


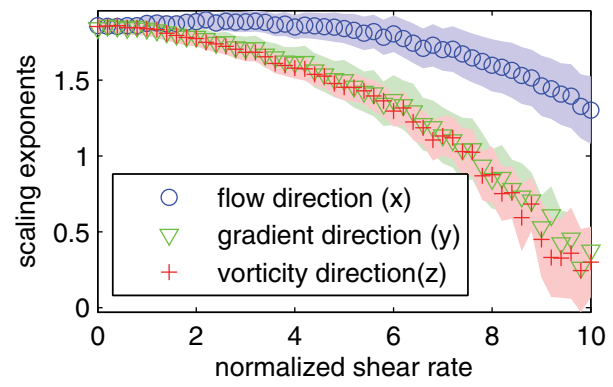

FIG. 4. Anisotropic effective conductivity exponents against normalized shear rate Pe. The effective scaling exponents are estimated from fitting (4.1) to the mean conductivity in each direction. A 95\% confident interval is shown as a shaded region around each exponent.

order in $\mathrm{Pe}$. However, a more complete finite size scaling analysis confirming the scaling over a wider range of volume fractions closer to the percolation threshold would be worthwhile to carry out (see, e.g., [31]). In the limit of small $P e$, the effective conductivity exponents increase with shear, and hence enhance the conductivity gains, agreeing with the phenomenon observed in experiments that a small amount of alignment of nanorods increases conductivity [7, 14]. As $P e$ increases to $O(1)$ and greater, the trends at small $P e$ reverse, and shear begins to diminish the effective conductivity exponents (and hence slow down and then reverse bulk conductivity gains with increasing shear rate). As the numerical fits to the exponents show, the scaling behaviors of the exponents are least sensitive in the flow direction. Overall, these results suggest there is an optimal bulk shear rate that maximizes the conductivity in each direction relative to the flow geometry.

4.3. Scaling of current distributions. In a previous study [41], we analyzed global features of the current distribution on a cubic lattice (i.e., bond percolation) and identified a robust, exponential large current tail that persists above threshold and is locally independent of the bond fraction. Here we perform similar analysis on the current distribution in physical 3D nanorod dispersions and show that similar scaling behavior arises in these more complex systems even though the centers of mass of the nanorods are random, the distances between nodes in the percolating clusters are highly nonuniformly distributed, and the percolating network is spatially anisotropic.

4.3.1. Robust exponential large current tail of current distributions. Figure 5 plots current distributions (left) and logarithmic current distributions (right) for isotropic $(P e=0)$ dispersions at various rod volume fractions $\theta$ above threshold $\theta_{c} \doteq 1.3 \%$, given a unit voltage source. Since the current distributions in all three directions are statistically similar as the rods are isotropically oriented, only the current distribution in the flow direction $(x)$ is shown. Let $f(i)$ be the probability density function (PDF) of the currents across the population of current-carrying edges (i.e., ignoring zero-current edges where present) and $h(x)$ be the corresponding PDF of the logarithmic current $X=\ln (I)$. The two distributions are related by $h(x)=f\left(e^{x}\right) e^{x}$.

First, the logarithmic transformation of current (Figure 5 (right)) exposes the small current region and reveals the power-law distribution of small currents, agreeing with what has been reported for bond percolation on a cubic lattice [47, 15, 16, 41]. For relatively large currents (i.e., to the right of the peak of the $h(x)$ distribution), 

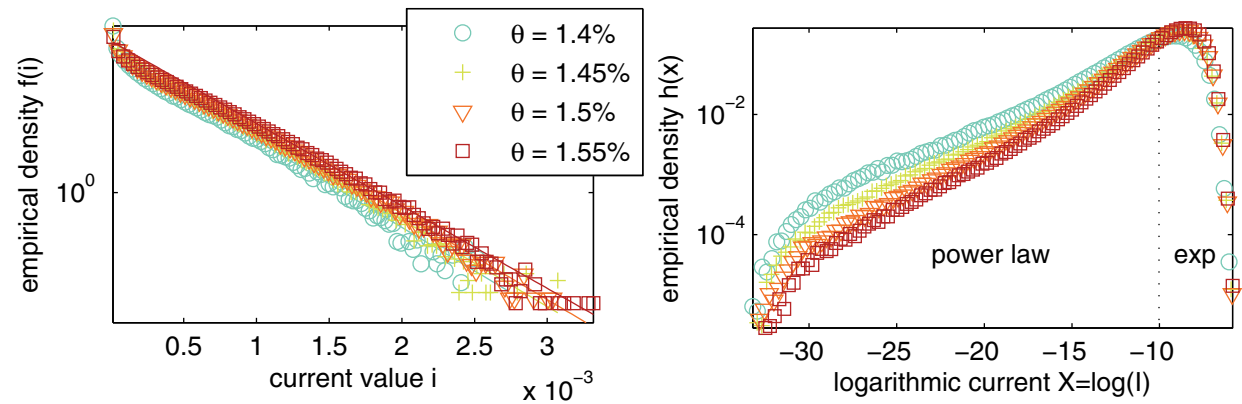

FIG. 5. Current distributions $f(i)$ (left) and logarithmic current distributions $h(x)$ (right) for isotropic $(P e=0)$ dispersions at various rod volume fractions $\theta$ given a unit voltage source. The logarithmic transformation of currents exposes small currents which have a power-law distribution (right panel) while the overall current distribution is exponential (left panel). The empirical densities are derived from the histograms of all the currents over 1000 realizations.
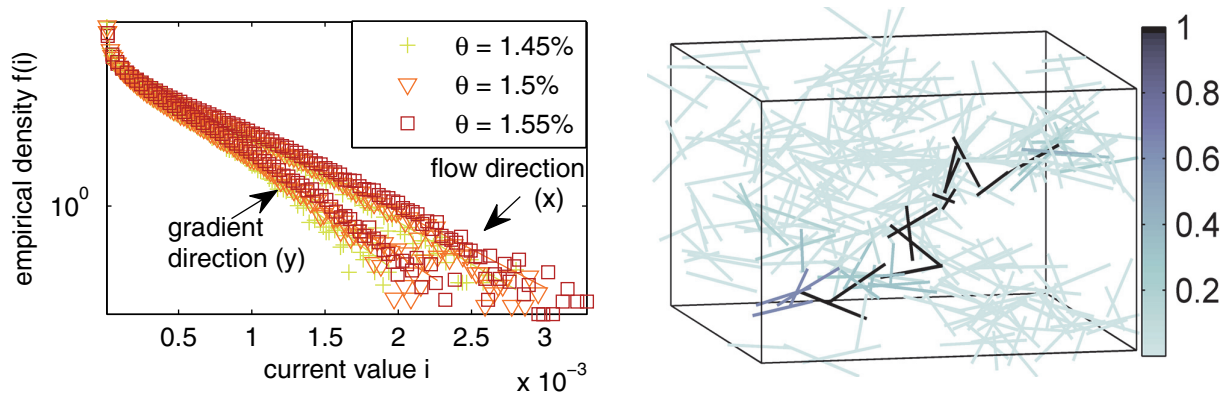

FIG. 6. Left: Current distributions in both the flow direction $(x)$ and the flow gradient direction (y) for sheared dispersions $(P e=5)$. The percolation threshold is $\theta_{c}(x) \doteq 1.35 \%$ in the flow direction (x) and $\theta_{c}(y) \doteq 1.4 \%$ in the flow gradient direction (y). Right: Visualization of the current-carrying rods and color-coded current values in a percolating cluster in the $x$ direction from one realization at $(P e, \theta)=(10, .015)$.

Figure 5 (left) is clearly suggestive of an exponential tail of the current distributions which expands broadly over large to moderate currents and dominates the current distribution at the expense of the vanishing power-law scaling in the small current tail.

The second conclusion drawn from Figure 5 is that the rate of the exponential tail above threshold is weakly dependent on the rod volume fraction $\theta$ near threshold given a unit voltage source, despite the fact that the current distribution close to the percolation threshold $\theta_{c} \doteq 1.3 \%$ shows some scattering because of the finite size effect. In [41] we quantitatively confirmed the independence of the exponential tail on bond density on cubic lattices and showed the convergence to a robust class of exponential distributions for the large current tail both near and above threshold.

In order to study the effect of shear, we plot the current distributions in both the flow direction $(x)$ and the flow gradient direction $(y)$ for sheared dispersions $(P e=5)$ in the left panel of Figure 6. We omit the current distributions in the vorticity direction $(z)$ for clarity because they almost overlap with the distributions in the $y$ direction. (In weak shear it is known that the principal axis of the rod orientational distribution is approximately along the 45 degree line in the shear plane [18].) Because of the shear-induced anisotropic rod orientation, the current distributions are 

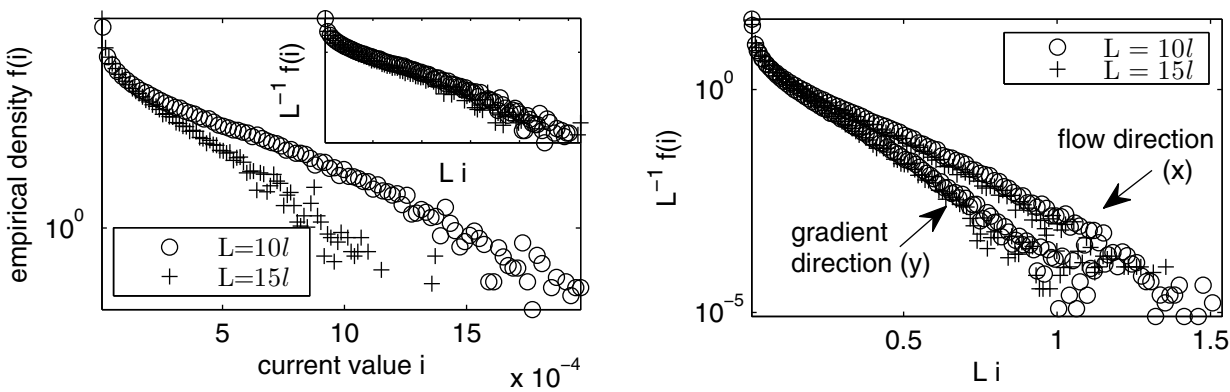

FIG. 7. Left: Current distributions in isotropic dispersions $(P e=0)$ at rod volume fraction $\theta=1.33 \%$. Two system sizes are considered: 10 times as long as a rod $(L=10 l)$ and 15 times as long as a rod $(L=15 l)$. The inset shows the same distributions rescaled by the system size $L$ with $u=1$ and $v=1$. Right: Rescaled current distributions in the flow direction $(x)$ and in the flow gradient direction $(y)$ in sheared $(P e=5)$ dispersions at rod volume fraction $\theta=1.5 \%$. It demonstrates that the finite size scaling form equation (4.3) holds in each direction under shear.

likewise anisotropic and the shear rate selects the exponential rates of the distributions. However, the robust exponential tail, which dominates the current distribution above threshold, persists in each spatial direction and is weakly dependent on the rod volume fraction $\theta$ as in the isotropic $(P e=0)$ case.

Mathematical justification for the existence of the exponential large current tail remains an open problem. Part of the problem appears to be that large currents are very rare while small currents are more abundant. We also note that the exponential cut-off in the cluster size distribution [46] may very well be related, as suggested by our colleague Michael Rubinstein, yet this remains an open problem. Importantly, the small numbers of large currents in the tail of the distribution of current-carrying rods exacerbate the separate phenomenon of there being relatively few current-carrying rods among the total dispersion, as remarked above (see the left panel of Figure 2). To illustrate the combined effect of small numbers of current-carrying rods and even smaller numbers of large currents, Figure 6 (right) visualizes the currents flowing in the $x$ direction in a single $\mathrm{MC}$ realization of a $3 \mathrm{D}$ sheared dispersion, at $(P e, \theta)=(10$, .015 ) and box length $L=250 \mathrm{~nm}$, demonstrating how very few of the approximately $10^{5}$ rods in this volume carry the largest currents. This result compels a further study of the stability of percolating components under small strain deformations, since such conditions arise in many thin film applications.

4.3.2. Finite size scaling analysis. Thus far we have considered the electrical response to a unit voltage source in a finite system of length $L$. To meaningfully describe the current distribution in an infinite system $(L \rightarrow \infty)$ and to better understand the effect of system size on the distribution, we perform a finite size scaling analysis as in [41]. Let $f_{L}(i)$ be the PDF of the current at system size $L$ for a unit voltage source. We propose a finite-size scaling form:

$$
L^{-u} f_{L}\left(L^{-v} i\right)=f^{\infty}(i),
$$

where $f^{\infty}$ is a function independent of $L$. By tuning $u$ and $v$ we aim to eliminate the effect of system size. Figure 7 (left) plots the current distributions in isotropic dispersions $(P e=0)$ at rod volume fraction $\theta=1.33 \%$ for different system sizes. The inset confirms that the PDFs for different system sizes collapse onto a single curve when $u=1$ and $v=1$. One can carry out the same argument at other rod volume 


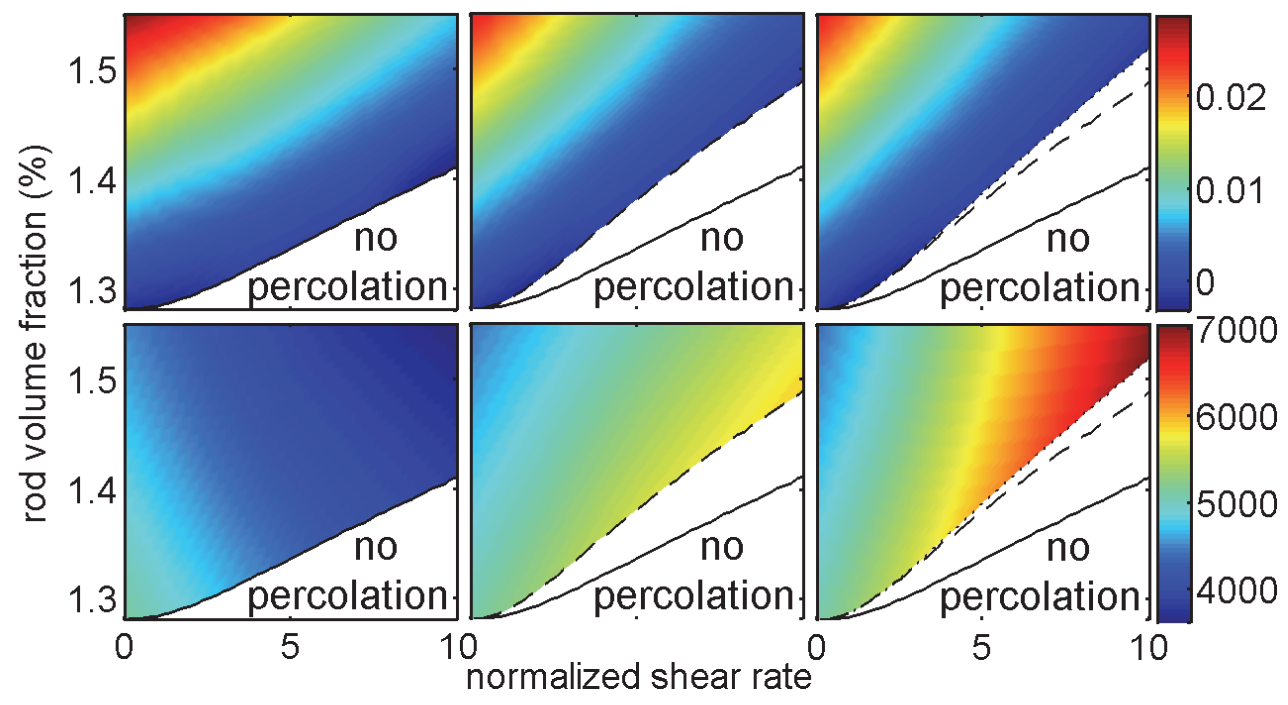

FIG. 8. Multiscale electrical properties across the percolation phase diagram (Figure 2 (right)). The top row shows the color-coded average bulk conductivities in the flow (x) direction (left panel), flow-gradient $(y)$ direction (center panel), and vorticity $(z)$ direction (right panel). The bottom row shows the rates of the exponential current tails in the three physical directions, respectively.

fractions above threshold $\theta_{c} \doteq 1.3 \%$ and find the same scaling exponents. In other words, $f^{\infty}$ can be viewed as the limiting current distribution for system size $L$ and external voltage source $V^{*}=L$ (i.e., a unit electric field). This simple scaling form indicates that $M_{k}$, the $k$ th moment of the current distribution, is a simple scaling function of $L$ :

$$
M_{k}=\int_{0}^{\infty} i^{k} f_{L}(i) d i=\int_{0}^{\infty} i^{k} L f^{\infty}(L i) d i \propto L^{-k}
$$

In [41] we showed that while this scaling of moments is not exact because of small currents which are multifractal, for large moments the exponential tail becomes dominant and (4.4) is a good approximation.

Again this simple finite size scaling form equation (4.3) remains the same in each direction under shear regardless of anisotropy of the current distribution, as shown in Figure 7 (right), which plots the rescaled current distributions $f^{\infty}=L^{-1} f_{L}\left(L^{-1} i\right)$ in both the flow direction $(x)$ and the flow gradient direction $(y)$ for a sheared $(P e=5)$ dispersion.

4.4. Electrical property phase diagrams. The above arguments show that the power-law scaling of bulk conductivities near threshold persists under shear, and further demonstrate a robust exponential tail of current distributions above threshold in response to a unit voltage source, even under shear. To conclude, we paint the average bulk conductivities and the rates of the exponential current tails onto the percolation phase diagram (Figure 2 (right)) in Figure 8. From left to right, the top three panels in the figure show the color-coded average bulk conductivities in the flow $(x)$ direction, flow gradient $(y)$ direction, and vorticity $(z)$ direction, respectively; and the bottom three panels show the rates of the exponential current tails in the three physical directions, respectively. The effect of shear on the conductivities is 
similar, while shear has relatively smaller impact in the flow $(x)$ direction. However, the exponential current tails present qualitatively different behaviors. In the flow $(x)$ direction, the exponential rate drops as shear increases, i.e., the tail becomes fatter, indicating that large currents are more frequent in the flow direction as shear increases. By contrast, in both the flow gradient $(y)$ and the vorticity $(z)$ directions the exponential rates increase with shear rate, meaning thinner tails, indicating that large currents are more rare in the plane normal to the flow direction as shear increases. This result is supported by asymptotic results [18] on the weak shear limit that show for small $P e$, the peak of the orientational PDF aligns with the $45^{\circ}$ axis in the shear plane $(x, y)$, and then progressively tilts toward the flow $(x)$ axis as $P e$ increases, always remaining in the shear plane for the low volume fractions relevant to percolation threshold. Thus the anisotropy in the large tail currents is strongly correlated with the orientational PDF of the rod phase.

Finally, we recall the scaling analysis from our recent paper [41] on random resistor networks in cubic bond percolation. From the established exponential large current tail across the percolation phase diagram (Figure 8, bottom row), two results immediately follow. First, the largest current in the network scales as $\ln (L)$. Note that previous studies on the largest current in lattice bond percolation [11, 32, 27, 33] do not analyze the tail of the current distribution, and there are arbitrarily many tails consistent with the logarithmic scaling of the maximum current. Knowing the tail is exponential, however, immediately implies the scaling of the maximum current. Second, we note the apparently weak dependence on volume fraction in the lower half of Figure 8, consistent with recent results on the geometrically simpler problem of random resistor networks on cubic lattices [41]. Indeed, other qualitative similarities with cubic random resistor networks then follow immediately (see again [41]), with the second moment of the current distribution relating to the bulk conductivity. Specifically, given a unit external voltage source, the bulk conductance $C$ is related to the current distribution by

$$
C / N=\sum_{b} i_{b}^{2} r_{b} / N=\int i^{2} r f(i, r) d i d r
$$

where the sum is taken over all edges with nonzero current, $i_{b}$ and $r_{b}$ are the current value and the resistance of edge $b$, respectively, and $N$ is the number of edges with nonzero current. Assuming that the current distribution and the edge resistance distribution are weakly dependent, the joint distribution $f(i, r)$ may be approximated by the product of individual distributions $f_{I}(i)$ and $f_{R}(r)$, and (4.5) then yields $C / N \approx\langle r\rangle \int_{0}^{\infty} i^{2} f_{I}(i) d i$. Since the second moment of the current distribution is dominated by the exponential large current tail, the approximate relationship of the bulk conductance with respect to rod volume fraction $\theta$ is revealed by the scaling behaviors of the exponential current tail to approximate the integral, the mean edge resistance, and the number of edges with large current. In other words, the observable bulk properties [e.g., (4.1) and (4.2)] can be reconstructed from particle-scale property distributions (e.g., the exponential large current tail of the current distribution and mean edge resistance). These observations are relevant especially to "active" composite materials where materials are exposed to mechanical loading and strain deformations.

5. Conclusion. In summary, we construct a network representation and apply associated network algorithms to calculate the linear electrical response on percolating, isotropic, and sheared, 3D nanorod dispersions. The methods are applied across 
the phase diagram of rod volume fraction and imposed normalized shear rate associated with a thin film flow. The 3D dispersions are generated from precomputed orientational probability distributions across the phase diagram [18, 17]. Network methods provide an efficient algorithm to identify the current-carrying rods in percolating components per 3D realization to accurately solve the linear electrical response per realization with particular gains in accuracy in the small current tail. MC calls to the orientational probability distributions at each point in the phase diagram deliver robust, multiresolution distributions of conductivity and current consistent with the statistical mechanics of flowing nanorod dispersions. Putting these tools together, we characterize the linear electrical response of sheared nanorod composite films over the dimensional percolation phase diagram of [54]. For each data point in the phase diagram, we determine the mean and variance of the bulk conductivity as well as current distributions within percolating rod clusters, with the ability to identify current values at the rod scale in each $3 \mathrm{D}$ physical realization.

The numerical results show that the celebrated power-law scaling of bulk conductivity above percolation threshold persists under shear, even while the conductivity becomes anisotropic due to the sheared nanorod orientational distribution. Furthermore, we show that the anisotropic power-law scaling in the observable bulk conductivity tensor is a consequence of a deeper transport property within the network. Namely, there is a robust exponential, large current tail in the current distribution; the exponential tails imply the conductivity scaling by taking second moments of the current distribution. We compute the large current tails, and their exponential rates, across the phase diagram of nanorod volume fraction and normalized shear rate. Remarkably, at approximately $1.3 \%$ rod volume fraction of aspect ratio 50 nanorods, the percolating dispersions typically yield mean conductivities that are only four orders of magnitude lower than the pure rod phase. The volume averaged boost in conductivity for the same parameters [54] is negligible compared to this percolation effect.

\section{REFERENCES}

[1] G. Ambrosetti, C. Grimaldi, I. Balberg, T. Maeder, A. Danani, and P. Ryser, Solution of the tunneling-percolation problem in the nanocomposite regime, Phys. Rev. B, 81 (2010), 155434.

[2] I. BalberG, Excluded-volume explanation of Archie's law, Phys. Rev. B, 33 (1986), pp. 36183620.

[3] I. Balberg, C. H. Anderson, S. Alexander, And N. Wagner, Excluded volume and its relation to the onset of percolation, Phys. Rev. B, 30 (1984), pp. 3933-3943.

[4] I. Balberg And N. Binenbaum, Computer study of the percolation threshold in a twodimensional anisotropic system of conducting sticks, Phys. Rev. B, 28 (1983), pp. 37993812.

[5] I. Balberg, N. Binenbaum, And N. Wagner, Percolation thresholds in the three-dimensional sticks system, Phys. Rev. Lett., 52 (1984), pp. 1465-1468.

[6] W. Bauhofer, S. C. Schulz, A. E. Eken, T. Skipa, D. Lellinger, I. Alig, E. J. Tozzi, And D. J. Klingenberg, Shear-controlled electrical conductivity of carbon nanotubes networks suspended in low and high molecular weight liquids, Polymer, 51 (2010), pp. 5024-5027.

[7] A. Behnam, J. Guo, And A. Ural, Effects of nanotube alignment and measurement direction on percolation resistivity in single-walled carbon nanotube films, J. Appl. Phys., 102 (2007), 044313.

[8] A. L. R. Bug, S. A. Safran, and I. Webman, Continuum percolation of rods, Phys. Rev. Lett., 54 (1985), pp. 1412-1415.

[9] A. Buldum and J. P. Lu, Contact resistance between carbon nanotubes, Phys. Rev. B, 63 (2001), 161403.

[10] G. A. Buxton And A. C. Balazs, Predicting the mechanical and electrical properties of nanocomposites formed from polymer blends and nanorods, Mol. Simulat., 30 (2004), 
pp. 249-257.

[11] S.-K. Chan, J. Machta, And R. A. Guyer, Large currents in random resistor networks, Phys. Rev. B, 39 (1989), pp. 9236-9239.

[12] L. De Arcangelis, S. Redner, and A. Coniglio, Anomalous voltage distribution of random resistor networks and a new model for the backbone at the percolation threshold, Phys. Rev. B, 31 (1985), pp. 4725-4727.

[13] M. DoI, Molecular dynamics and rheological properties of concentrated solutions of rodlike polymers in isotropic and liquid crystalline phases, J. Polymer Sci. Polymer Phys. Ed., 19 (1981), pp. 229-243.

[14] F. DU, J. E. Fischer, AND K. I. Winey, Effect of nanotube alignment on percolation conductivity in carbon nanotube/polymer composites, Phys. Rev. B, 72 (2005), 121404.

[15] E. Duering and D. J. Bergman, Current distribution on a three-dimensional, bond-diluted, random-resistor network at the percolation threshold, J. Statist. Phys., 60 (1990), pp. 363381.

[16] E. Duering, R. Blumenfeld, D. J. Bergman, A. Aharony, and M. Murat, Current distributions in a two-dimensional random-resistor network, J. Statist. Phys., 67 (1992), pp. 113-121.

[17] M. G. Forest, Q. Wang, And R. Zhou, The flow-phase diagram of Doi-Hess theory for sheared nematic polymers II: Finite shear rates, Rheol. Acta, 44 (2004), pp. 80-93.

[18] M. G. Forest, Q. WAng, And R. Zhou, The weak shear kinetic phase diagram for nematic polymers, Rheol. Acta, 43 (2004), pp. 17-37.

[19] M. G. Forest, X. Zheng, R. Zhou, Q. Wang, and R. Lipton, Anisotropy and dynamic ranges in effective properties of sheared nematic polymer nanocomposites, Adv. Funct. Mater., 15 (2005), pp. 2029-2035.

[20] B. P. GRAdY, Recent developments concerning the dispersion of carbon nanotubes in polymers, Macromol. Rapid Comm., 31 (2010), pp. 247-257.

[21] G. R. Grimmett, Percolation, 2nd ed., Springer, Berlin, 1999.

[22] N. Grossiord, J. Loos, O. Regev, and C. E. Koning, Toolbox for dispersing carbon nanotubes into polymers to get conductive nanocomposites, Chem. Mater., 18 (2006), pp. 1089-1099.

[23] M. Grujicic, G. CaO, AND W. N. Roy, A computational analysis of the percolation threshold and the electrical conductivity of carbon nanotubes filled polymeric materials, J. Mater. Sci., 39 (2004), pp. 4441-4449.

[24] A. A. Gusev and H. R. Lusti, Rational design of nanocomposites for barrier applications, Adv. Mater., 13 (2001), pp. 1641-1643.

[25] A. A. Gusev and M. G. Rozman, Numerical search for morphologies providing ultra high elastic stiffness in filled rubbers, Computat. Theor. Polymer Sci., 9 (1999), pp. 335-337.

[26] S. Hess, Fokker-Planck-equation approach to flow alignment in liquid crystals, Z. Naturforsch. Teil A, 31 (1976), pp. 1034-1037.

[27] B. KAhng, G. G. BATROUnI, AND S. Redner, Logarithmic voltage anomalies in random resistor networks, J. Phys. A, 20 (1987), pp. L827-L834.

[28] S. B. Kharchenko, J. F. Douglas, J. Obrzut, E. A. Grulke, and K. B. Migler, Flowinduced properties of nanotube-filled polymer materials, Nat. Mater., 3 (2004), pp. 564-568.

[29] G. Kwon, Y. Heo, K. Shin, And B. J. Sung, Electrical percolation networks of carbon nanotubes in a shear flow, Phys. Rev. E, 85 (2012), 011143.

[30] C. Li, E. T. Thostenson, And T.-W. Chou, Dominant role of tunneling resistance in the electrical conductivity of carbon nanotube-based composites, Appl. Phys. Lett., 91 (2007), 223114.

[31] J. Li And S.-L. Zhang, Conductivity exponents in stick percolation, Phys. Rev. E, 81 (2010), 021120.

[32] Y. S. Li And P. M. Duxbury, Size and location of the largest current in a random resistor network, Phys. Rev. B, 36 (1987), pp. 5411-5419.

[33] J. Machta And R. A. Guyer, Largest current in a random resistor network, Phys. Rev. B, 36 (1987), pp. 2142-2146.

[34] G. W. Milton, The Theory of Composites, Cambridge University Press, Cambridge, UK, 2002.

[35] M. Moniruzzaman and K. I. Winey, Polymer nanocomposites containing carbon nanotubes, Macromolecules, 39 (2006), pp. 5194-5205.

[36] S. H. Munson-McGee, Estimation of the critical concentration in an anisotropic percolation network, Phys. Rev. B, 43 (1991), pp. 3331-3336.

[37] C.-W. Nan, Y. Shen, And J. Ma, Physical properties of composites near percolation, Annu. Rev. Mater. Res., 40 (2010), pp. 131-151.

[38] J. Obrzut, J. F. Douglas, S. B. Kharchenko, and K. B. Migler, Shear-induced conductorinsulator transition in melt-mixed polypropylene-carbon nanotube dispersions, Phys. Rev.

Copyright (c) by SIAM. Unauthorized reproduction of this article is prohibited. 
B, 76 (2007), 195420.

[39] Z. Ounaies, C. Park, K. E. Wise, E. J. Siochi, and J. S. Harrison, Electrical properties of single wall carbon nanotube reinforced polyimide composites, Compos. Sci. Tech., 63 (2003), pp. 1637-1646.

[40] R. H. Schmidt, I. A. Kinloch, A. N. Burgess, and A. H. Windle, The effect of aggregation on the electrical conductivity of spin-coated polymer/carbon nanotube composite films, Langmuir, 23 (2007), pp. 5707-5712.

[41] F. Shi, S. Wang, M. G. Forest, and P. J. Mucha, Percolation-induced exponential scaling in the large current tails of random resistor networks, Multiscale Model. Simul., 11 (2013), pp. $1298-1310$.

[42] J. Silva, R. Simoes, S. Lanceros-Mendez, and R. Vaia, Applying complex network theory to the understanding of high-aspect-ratio carbon-filled composites, Europhys. Lett., 93 (2011), 37005.

[43] R. Simoes, J. Silva, R. Vaia, V. Sencadas, P. Costa, J. Gomes, and S. Lanceros-Mendez, Low percolation transitions in carbon nanotube networks dispersed in a polymer matrix: Dielectric properties, simulations and experiments, Nanotechnology, 20 (2009), 035703.

[44] T. Skipa, D. Lellinger, W. Böhm, M. Saphiannikova, and I. Alig, Influence of shear deformation on carbon nanotube networks in polycarbonate melts: Interplay between buildup and destruction of agglomerates, Polymer, 51 (2010), pp. 201-210.

[45] D. Stauffer, J. Adler, And A. Aharony, Universality at the three-dimensional percolation threshold, J. Phys. A, 27 (1999), pp. L475-L480.

[46] D. Stauffer and A. Aharony, Introduction to Percolation Theory, CRC Press, Boca Raton, FL, 1994.

[47] J. P. Straley, Current distribution in random resistor networks, Phys. Rev. B, 39 (1989), pp. 4531-4535.

[48] G. Strang, Introduction to Applied Mathematics, Wellesley-Cambridge Press, Wellesley, MA, 1986.

[49] C. Veerman, L. M. C. Sagis, P. Venema, and E. van der Linden, The effect of shear flow on the percolation concentration of fibrillar protein assemblies, J. Rheol., 49 (2005), pp. 355-368.

[50] S. I. White, P. M. Vora, J. M. Kikkawa, And K. I. Winey, Resistive switching in bulk silver nanowire-polystyrene composites, Adv. Funct. Mater., 21 (2011), pp. 233-240.

[51] H.-P. XU AND Z.-M. DANG, Electrical property and microstructure analysis of poly(vinylidene fluoride)-based composites with different conducting fillers, Chem. Phys. Lett., 438 (2007), pp. 196-202.

[52] J. Xu, W. Florkowski, R. Gerhardt, K.-S. Moon, and C.-P. Wong, Shear modulated percolation in carbon nanotube composites, J. Phys. Chem. B, 110 (2006), pp. 12289-12292.

[53] X. Zheng, M. G. Forest, R. Lipton, R. Zhou, And Q. Wang, Exact scaling laws for electrical conductivity properties of nematic polymer nanocomposite monodomains, Adv. Funct. Mater., 15 (2005), pp. 627-638.

[54] X. Zheng, M. G. Forest, R. Vaia, M. Arlen, and R. Zhou, A strategy for dimensional percolation in sheared nanorod dispersions, Adv. Mater., 19 (2007), pp. 4038-4043.

[55] H. Zhou, M. G. Forest, X. Zheng, Q. Wang, and R. Lipton, Extension-enhanced conductivity of liquid crystalline polymer nano-composites, Macromol. Symp., 228 (2005), pp. 81-90.

Copyright $@$ by SIAM. Unauthorized reproduction of this article is prohibited. 